\title{
TEGRETOL NO TRATAMENTO DA EPILEPSIA
}

\author{
Jorge Armrrust-Figueiredo *
}

O iminostilbene (Tegretol $* *$ ) é uma droga relativamente nova que tem sido usada principalmente no tratamento da neuralgia do trigêmeo e que possui acentuada atividade anticonvulsivante. Sua estrutura é 5-carbamil-5H-dibenzo-b,f-azepina, que se assemelha à da imipramina (Tofranil, Insidon).

Sua ação anticonvulsivante foi demonstrada experimentalmente por Herhandez-Peon ${ }^{9}$ em 1962, e ulteriormente confirmada na clínica por diferentes autores. A apreciação geral dos resultados já publicados demonstra que a ação do Tegretol é melhor evidenciada nas crises psicomotoras e grande mal e na epilepsia temporal. Ao lado da ação anticonvulsivante, o Tegretol possui efeito psicotrópico, melhorando a depressão psíquica, favorecendo a atividade mental e aumentando a atenção.

Jongmans ${ }^{10}$ usou a droga no tratamento de 70 pacientes, dos quais 43 apresentavam crises G.M., 3 crises P.M. e 24 crises psicomotoras. Observou resposta favorável em 24 pacientes com crises G.M. e em 10 com crises psicomotoras. Sigwald e col. ${ }^{14}$ estudaram 160 pacientes, relatando resultados favoráveis em cêrca de dois têrços dos casos; salientam que as melhores respostas foram observadas em pacientes com crises psicomotoras e que a droga é pouco eficaz nos casos de pequeno mal. Arieff e Mier ${ }^{1}$ trataram 26 pacientes com crises G.M. e/ou psicomotoras, referindo resultados satisfatórios em 10 (39\%), nos quais o contrôle das crises foi duradouro ou temporário. Bonduelle e col. ${ }^{5}$ estudaram 100 pacientes com crises de diferentes tipos (G.M., P.M., psicomotoras e focais), de aparecimento isolado ou associado; observaram resposta favorável em 69, particularmente entre aqueles que apresentavam crises G.M. ou psicomotoras isoladas. Madalena 12 referiu $47 \%$ de remissão completa em 34 pacientes com crises G.M. Tchicaloff e Pennatti ${ }^{15}$ usaram a droga em 18 pacientes com crises G.M. ou psicomotoras e epilepsia temporal, verificando resultados bons em $11(61 \%)$.

\section{MATERIAL E METODOS}

\footnotetext{
Estudamos 68 pacientes com epilepsia e cujas crises nāo haviam sido controladas pelo emprêgo de diferentes esquemas terapêuticos. Dos 68 pacientes, 32 eram do sexo masculino e 36 do sexo feminino e suas idades variaram entre 12 e 37 anos. A incidencia de tipos de crises foi: focais temporais (psicomotoras 42 ; complexas 12); focais não temporais 11 ; centrencefálicas (PM) 3 .
}

Departamento de Neurologia da Faculdade de Medicina de Ribeirão Prêto, da Universidade de São Paulo: * Professor Catedrático.

** Tegretol, fornecido por Geigy do Brasil S.A. 
Todos os pacientes haviam sido tratados anteriormente no Departamento de Neurologia da Faculdade de Medicina de Ribeirão Prêto, durante, pelo menos, 10 meses consecutivos, e não haviam respondido favoràvelmente ao tratamento com diferentes drogas anticonvulsivantes. Todos tinham sido submetidos a um ou mais exames eletrencefalográficos antes do inicio do tratamento com Tegretol e 65 tiveram seu traçado repetido durante o periodo de observação, que foi, no minimo de 11 meses.

o seguimento dos doentes foi feito em ambulatório. De início, o Tegretol foi acrescentado à medicação pré-existente; progressivamente sua dosagem foi aumentada, com redução paralela das demais drogas, até que a substituição fôsse completada. Em todos os casos, no entanto, a administração de um barbitúrico foi mantida durante todo o tratamento, de acôrdo com a nossa orientação na terapêutica da epilepsia ${ }^{3}$.

A dosagem da medicação variou de acôrdo com a idade do paciente e com as necessidades individuais. Na maioria dos casos, porém, usamos $600 \mathrm{mg}$ diários, em doses fracionadas ( 1 comprimido 3 vêzes ao dia). A dose máxima empregada foi de $1.000 \mathrm{mg}$ diários, em 1 caso; 3 pacientes receberam $800 \mathrm{mg}$ diários durante periodos de 6 a 13 meses; 17 não necessitaram doses superiores a $400 \mathrm{mg}$ diários.

\section{RESULTADOS}

Para a avaliação do valor do Tegretol consideramos quatro graus de resultados: muito bom, quando houve desaparecimento das manifestações clínicas por um prazo mínimo de 10 meses; bom, quando surgiram raras crises isoladas ou quando houve redução numérica das crises superior a $60 \%$; regular, quando a redução daes crises foi inferior a $60 \%$; nulo ou piora da frequência ou intensidade das crises clínicas.

Os resultados conseguidos nos 68 pacientes foram bastante satisfatórios (quadro 1). Dos 54 pacientes que apresentavam epilepsia focal de projeção temporal, $27(50 \%)$ apresentaram resposta muito boa ao tratamento e 19 (95\%) resposta boa; em apenas 8 pacientes (15\%) a redução das crises foi inferior a $60 \%$. Neste mesmo grupo, os pacientes com crises psicomotoras obtiveram melhor resultado que aqueles com crises mistas.

Dos 11 casos de epilepsia focal de projecão não temporal, $45 \%$ ficaram livres de suas crises durante o período de seguimento, $36 \%$ apresentaram resultado bom e $19 \%$ resultado regular.

Nestes dois grupos de pacientes, a resposta favorável ao tratamento (incluindo-se os resultados muito bom e bom) alcancou o valor de $82 \%$.

Por outro lado, a medicação nāo demonstrou ser de valor no tratamento das crises de tipo pequeno mal; em um caso houve discreta redução do número de crises, mas dois pacientes tiveram agravamento do quadro clinico. Nestes 3 casos, o Tegretol foi suspenso logo que verificamos a sua ineficácia.

Em relação aos efeitos psicotrópicos da droga, observamos ação favorável sôbre as manifestaçôes de ordem psíquica.

Dos 68 pacientes, 49 apresentavam algum distúrbio de tipo: (a) bradipsiquismo, (b) irritabilidade ou agressividade e (c) angústia e depressão. Com freqüência, tais manifestações apareciam associadas, traduzindo-se por distúrbios de comportamento familiar e/ou social (escolar ou profissional). Dêstes 49 pacientes, 46 pertenciam ao grupo de epilepsia temporal e 3 ao grupo de epilepsia focal nāo temporal.

$O$ uso do medicamento trouxe acentuada melhora dessas manifestaçoes na grande maioria dos casos; 33 pacientes ficaram pràticamente livres das manifestações, 11 apresentavam melhoras evidentes e os demais não mostraram alteração do quadro psíquico. Dos 49 pacientes, 34 tinham crises psicomotoras, 8 crises mistas e 14 crises focais não temporais. 


\begin{tabular}{|c|c|c|c|c|c|c|c|c|c|}
\hline \multirow{3}{*}{ Tipo de Epilepsia } & \multirow{3}{*}{$\begin{array}{c}N \cdot^{o} \\
\text { de } \\
\text { casos }\end{array}$} & \multicolumn{8}{|c|}{$R e s u l t a d o s$} \\
\hline & & \multicolumn{2}{|c|}{$\begin{array}{c}\text { Muito } \\
\text { bom }\end{array}$} & \multicolumn{2}{|c|}{ Bom } & \multicolumn{2}{|c|}{ Regular } & \multicolumn{2}{|c|}{$\begin{array}{c}\text { Nulo } \\
\text { ou piora }\end{array}$} \\
\hline & & $n .0$ & $\%$ & $n .^{\circ}$ & $\%$ & $n .^{o}$ & $\%$ & $n . "$ & $\%$ \\
\hline$=$ & & & & & & & $=$ & & $=$ \\
\hline Focal temporal: & 54 & 27 & 59 & 19 & 35 & 8 & 15 & 0 & 0 \\
\hline crises psicomotoras & 42 & 23 & 55 & 13 & 30 & 6 & 15 & 0 & 0 \\
\hline crises complexas & 12 & 4 & 33 & 6 & 50 & 2 & 17 & 0 & 0 \\
\hline Focal não temporal & 11 & 5 & 45 & 4 & 33 & 2 & 19 & 0 & 0 \\
\hline Centrencefálica (PM) & 3 & 0 & 0 & 0 & 0 & 1 & 33 & 2 & 67 \\
\hline Total & 68 & 32 & 47 & 23 & 34 & 11 & 16 & 2 & 3 \\
\hline
\end{tabular}

Quadro 1 - Resultados obtidos com o uso de Tegretol em 68 pacientes epilépticos.

A comparação dos achados eletrencefalográficos de cada paciente, antes e após o inicio do tratamento, mostrou melhora da atividade elétrica anormal em $27(42 \%)$ pacientes, enquanto que nos $38(58 \%)$ restantes os traçados permaneceram inalterados. Nos 3 pacientes com crises P.M. os exames de contrôle não foram realizados, dada a suspensão do medicamento.

As reações secundárias foram discretas, tendo sido referidas por 23 pacientes. Sensação de "tontura" ou "mal estar" surgiu em 8 pacientes, dentre os quais 4 recebiam $800 \mathrm{mg}$ ou mais da droga; dois pacientes se queixaram de náuseas, três de cefaléia e dois de erupção cutânea. Um dêstes dois pacientes teve discreta diminuição do número de leucócitos que foi ràpidamente controlada com a suspensão temporária da droga.

\section{COMENTARIOS}

A avaliação geral dos resultados mostra que o Tegretol é medicamento de real valor no tratamento da epilepsia focal. Em nosso material, respostas bastante favoráveis foram obtidas em $82 \%$ dos casos, cujo seguimento foi, no mínimo, de 11 meses. Tal cifra, é ligeiramente superior àquelas registradas na literatura e que correspondem, em geral, a dois têrços dos casos tratados $5,6,10,14,15$.

Embora o número de casos de epilepsia centrencefálica com crises P.M. tenha sido muito reduzido em nossa investigação, os resultados foram totalmente insatisfatórios.O mesmo não ocorreu na casuística de Bonduelle e col. ${ }^{5}$ que obtiveram resultados que consideraram como bons em 8 dentre 16 casos; devemos salientar, no entanto, que em 6 dêstes pacientes, o Tegretol foi associado às dionas que, isoladas, não vinham controlando as crises clínicas. Os demais autores não referem efeito benéfico da droga nas crises P.M. 1, 5, 10, 11 .

Fator importante na avaliação geral dos resultados é a ação psicotrópica do medicamento. Houve influência favorável em todos os pacientes que mostravam algum distúrbio de tipo psíquico. Tal verificação já havia sido feita, em maior ou menor grau, pela maioria dos autores $1,4,5,7,10,11$, $12,13,14,15$. 
Tal qualidade do Tegretol assume importância capital, pois o contrôle dêsse tipo de manifestações representava a maior dificuldade para o tratamento. Em elevada freqüência os pacientes submetidos à terapêutica anticonvulsivante habitual, apresentam redução de suas crises motoras, inclusive dos automatismos, mas a piora dos fenômenos psíquicos é quase a regra. O comportamento familiar se agrava, o rendimento profissional ou escolar decai de maneira acentuada, o bradipsiquismo se torna mais evidente e a depressão e a angústia se intensificam. A associação de outras drogas se torna obrigatória em muitos casos e os resultados nem sempre são animadores. Ao que parece, com o uso do Tegretol tal inconveniente pode ser obviado.

A ação do Tegretol sôbre a atividade elétrica cerebral não pôde ser estabelecida no presente estudo. Houve melhora do traçado em $42 \%$ dos casos, mas a evolução eletrencefalográfica não se faz paralelamente ao estudo clínico. Achado semelhante é apresentado por outros autores 1, 5, 12, 14 .

A tolerância ao medicamento foi bôa; discretos sinais secundários surgiram em alguns pacientes. Em dois dêles foram observadas manifestações cutâneas alérgicas, sendo que em um houve queda transitória da taxa de leucócitos. Fenômenos tóxicos discretos foram também assinalados por outros autores $5,6,7,10,11,12,14$.

Convém assinalar que o limite de segurança da dosagem é bastante amplo. Arieff e Mier ${ }^{1}$ salientam que doses de $2.600 \mathrm{mg}$ diários tem sido administradas, sem ocasionar reações secundárias graves. Contudo, é preciso referir que o uso prolongado da droga, por periodos superiores a um ou dois anos, pode ser responsável pelo aparecimento de distúrbios de acomodação visual, de leucopenia, eosinofilia, oliguria com hipertensão arterial, e púrpura. Um caso fatal foi citado por Arieff e Mier ${ }^{1}$ provocado por trombocitopenia.

Por outro lado, Hajnsek e Sartorius ${ }^{8}$ referem um caso de intoxicação grave por Tegretol; um paciente ingeriu 100 comprimidos, equivalentes a $20 \mathrm{~g}$, mas sobreviveu. Surgiram sinais neurológicos e psiquiátricos difusos, mas os exames de laboratório não evidenciaram lesões hepáticas, renais ou de medula óssea; ocorreu leucopenia transitória, mas a paciente pôde deambular ao fim de uma semana.

De nossos resultados podemos concluir: 1) o Tegretol tem ação favorável nas epilepsias focais, reduzindo de modo sensível as manifestaçōes clínicas; 2) as crises psicomotoras, motoras ou mistas são igualmente influenciadas; 3) a ação psicotrópica é evidente, reduzindo as manifestações psíquicas e permitindo melhor ambientação do paciente na família e nas atividades profissionais ou escolares; 4) a influência sôbre as alterações eletrencefalográficas foi verificada em pouco menos de metade dos casos; 5) a tolerância à droga é boa, tendo surgido apenas discretas manifestações de tipo tóxico; 6) a dosagem útil, para nossos casos, foi reduzida e bastante inferior ao limite considerado como de segurança; 7) o Tegretol não tem valor no contrôle das crises tipo pequeno mal. 


\section{RESUMO}

O Tegretol (5-carbamil-5H-dibenzo-b,L-azepina) foi usado no tratamento de 68 pacientes com epilepsia; 54 apresentavam epilepsia temporal (42 com crises psicomotoras e 12 com crises complexas), 11 epilepsia focal de projeção não temporal e 3 epilepsia centrencefálica com crises P.M. Resultados satisfatórios foram observados em $82 \%$ dos 65 pacientes com epilepsia focal; os 3 pacientes com crises P.M. não foram influenciados pelo tratamento.

A ação sôbre as manifestações psíquicas que acompanhavam o quadro crítico ou intercrítico das crises ficou bem evidenciada. Os pacientes melhoraram de seus sintomas na grande maioria dos casos, conseguindo adaptar-se melhor no ambiente familiar, profissional e escolar.

O efeito sôbre as alterações eletrencefalográficas é moderado, e a evolução clínica não acompanha as modificações dos traçados.

As doses usadas, em geral de 400 a $600 \mathrm{mg}$ diários, são suficientes para o contrôle das crises na maioria dos casos e estão muito abaixo dos limites considerados como de segurança. As reações secundárias foram observadas em pequeno número de casos e foram sempre transitórias.

O Tegretol representa, em nossa opinião, recurso de valor no trataemnto das epilepsias focais, particularmente nas crises psicomotoras.

\section{SUMMARY}

Tegretol in the treatment of epilepsy

Tegretol (5-carbanyl-5H-dibenzo-b,L-azepina) was used experimentally in the theatment of 68 epileptic patients; 54 had temporal lobe epilepsy ( 42 had psychomotor and 12 had complex seizures), 11 had focal non-temporal lobe epilepsy and 3 had P.M. epilepsy.

Satisfactory results were obtained in 82 per cent of the 65 patients with focal epilepsy, but failure of the treatment was observed in all cases of P.M. epilepsy.

The drug has a very good influence on the critical and intercritical mental phenomena which are frequently present in these patients. The personality of the patient improves and he becomes better adjusted to his family, his work or school.

The action of the electroencephalogram is not very clear. Some records were improved, others remained inaltered.

The dosage used varied from 400 to $600 \mathrm{mg}$ daily in the great majority of cases. Side effects were not common and not severe. One patient developed transitory drop in leukocytes. 
Tegretol is a drug with anticonvulsivant and psychotropic properties, and has a very pronounced effect on focal epilepsy particularly on psychomotor seizures.

\section{REFERENCIAS}

1. ARIEFF, A. J. \& MIER, M. - Anticonvulsant and psychotropic action of Tegretol. Neuroly 16:107-110, 1966.

2. ARMBRUST-FIGUEIREDO, J. - Contribuição ao estudo clínico da epilepsia temporal. Escolas Profissionais Salesianas, São Paulo, 1958.

3. ARMBRUST-FIgUEIREDO, J. - A Metisergide no tratamento da epilepsia temporal. Arq. Neuro-Psiquiat. (São Paulo) 25:221-226, 1967.

4. Bonduelle, M.; Bouygues, P.; SAllou, C. \& Chemally, R. - Bilan de l'experimentation clinique de l'anti-épileptique G-32.883: résultats de 89 observations. III Congr. Int. Neuropsychopharmacol., Munique, 1962.

5. Bonduelle, M.; BouYgues, P.; SAllou, C, \& GRobuis, S. - Expèrimentation clinique de l'anti-épileptique G-32.883 (Tégretol). Rev. Neurol. 110: 209-215, 1964.

6. DAVIS, E. - Clinical evaluation of a new anticonvulsant, G-32.883. Med. J. Aust. 1:150-152, 1964.

7. HAGEN, K. \& RIGHTER, U. - Therapeutische Erfahrungen mit einem neuartigen Antiepilepticum bei kindern und Jugendlichen. Nervenarzt 35:312-314, 1964.

8. HAJNSSEK, F. \& SARTORIUS, N. - A case of intoxication with Tegretol. Epilepsia (Amsterdam) 5:371-375, 1964.

9. HERNANDEZ-PEON, R. - Anticonvulsive action of G-32.883. III Congr. Int. Neuropharmacol., Munique, 1962.

10. JONGMANS, J. W. M. - Report on the anti-epileptic action of Tegretol. Epilepsia (Amsterdam) 5:74-82, 1964.

11. LORGE, M. - Klinische Erfahrungen mit einen neuen Antiepilepticum, Tegretol (G-32.883), mit besonderer Wirkung auf die epileptische Wesenveranderung. Schweiz. Med. Wschr. 93:1042-1047, 1963.

12. MADALENA, J. C. - Um nôvo psicotropo anticonvulsivante, G-32.883. Hospital (Rio de Janeiro) 67:731-743, 1965.

13. MULLER, H. A. - Ein neuartiges Antiepilepticum bei chronisch anstalsbedurftigen Epileptikern. Nervenarzt 34:463-464, 1963.

14. SIGWALD, J.; BONDUELLE, M.; SALlOU, C. R.; RAVERDY, P. H. \& van STEENBRUdGE, A. - Un nouveau anti-épileptique. Presse méd. (Paris) 72:2323-2324, 1964.

15. TCHICALOFF, M. \& PENNETTI, F. - Résultats thérapeutiques d'un nouvel anti-épileptique, le Tégertol. Schweiz. Med. Wschr. 93:1664-1666, 1963.

Departamento de Neurologia - Faculdade de Medicina - Caixa Postal 301 Ribeirão Prêto, SP - Brasil. 